\title{
Towards SERS-based multiplexed monitoring of protease activity using non-natural aromatic amino acids
}

\author{
Nina Turk ${ }^{1,2, *}$, Hans Demol ${ }^{3,4}$, Andre Skirtach ${ }^{2,5}$, Roel Baets ${ }^{1,2}$ and Kris Gevaert ${ }^{3,4}$ \\ ${ }^{1}$ Ghent University - IMEC, Photonics Research Group, Technologiepark 126, 9052 Ghent, Belgium \\ ${ }^{2}$ Center for Nano- and Biophotonics, Ghent, Belgium \\ ${ }^{3}$ VIB-UGent Center for Medical Biotechnology, Ghent, Belgium \\ ${ }^{4}$ Ghent University, Department of Biomolecular Medicine, Belgium \\ ${ }^{5}$ Ghent University, Department of Biotechnology, Ghent, Belgium
}

\begin{abstract}
Surface Enhanced Raman Spectroscopy (SERS) allows sensitive and selective detection of protease activity by monitoring the cleavage of specific peptide substrates. Furthermore, it offers the possibility for multiplexing, during which the activity of two (or more) proteases with different specificities is detected simultaneously. To distinguish between the contributions of different proteases, different aromatic amino acids with non-overlapping SERS peaks need to be used as Raman reporters. As the three natural aromatic amino acids only offer limited possibilities for multiplexing, we examined several non-natural aromatic amino acids with the aim of expanding multiplexing possibilities. We recorded their SERS spectra for the Raman shifts of 300-1700 $\mathrm{cm}^{-1}$ and identified their characteristic SERS peaks. Of the examined nonnatural aromatic amino acids, 3-nitro-tyrosine and two phenylalanines containing stable heavy isotopes seem particularly promising for multiplexing applications. Besides exhibiting characteristic SERS peaks in the spectral region of interest, these non-natural aromatics provide strong SERS peaks compared to natural aromatic amino acids, consequently improving detection sensitivity.
\end{abstract}

Raman spectroscopy allows for highly specific detection of molecules based on their vibrational modes. Raman signals are however inherently weak, but SurfaceEnhanced Raman Spectroscopy (SERS) can be employed to enhance such weak Raman signals near the surface of plasmonic nanostructures. Among others, SERS can be used for sensitive and selective detection of protease activity using gold nanodomes to achieve the SERS enhancement [1]. Proteases are enzymes that catalyse the hydrolysis of peptide bonds and, amongst others, these enzymes play important roles in various human diseases [2]. Many proteases cleave their peptide substrates specifically and by incorporating two aromatic amino acids as Raman reporters in the peptide substrate with the protease cleavage site separating these aromatic amino acids, SERS can be used to monitor protease activity as schematically shown in Figure 1.
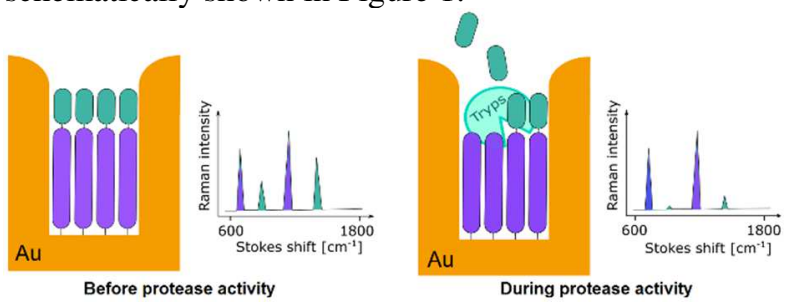

Fig. 1. The concept of protease activity detection via peptide cleavage using surface-enhanced Raman spectroscopy. The peptide forms a monolayer on the gold nanostructures, and is then cleaved by the protease trypsin (Tryps).
SERS also holds great potential for multiplexing, this to simultaneously detect the activity of two (or more) proteases with different specificities. To achieve this, it is crucial that one can distinguish the contributions of different proteases by using aromatic amino acids with non-overlapping SERS peaks. Since the three natural aromatic amino acids, namely phenylalanine, tyrosine and tryptophan, offer only limited opportunities for multiplexing measurements, we turned to non-natural aromatic amino acids. We investigated selected nonnatural amino acids for non-overlapping SERS peaks to expand the possibilities of multiplexing. Such non-natural aromatics can additionally offer potentially higher Raman cross-sections, which provide higher SERS signal strengths than those of natural aromatic, thus improving the signal-to-noise ratio. In this paper, we report on the SERS spectra and SERS peaks of different aromatic amino acids and assess their suitability for multiplexing applications.

\section{Experiments and results}

We investigated all three natural and eight selected nonnatural aromatic amino acids (Table 1). We have chosen to investigate several derivatives of tyrosine [3] and phenylalanine [4], as well as phenylalanine molecules containing stable heavy isotopes of hydrogen, carbon and nitrogen [5]. We incorporated the aromatic acids in a

* Corresponding author: nina.turk@ugent.be 
peptide chain $\mathrm{NH}_{2}-\mathrm{CALNNXG-COOH}$, where $\mathrm{X}$ denotes the individual aromatic amino acid. In this peptide sequence, cysteine $(\mathrm{C})$ allows the peptide to bind to the gold nanodomes, which provide the SERS enhancement [1]. The SERS spectra were obtained using a laser power of $1 \mathrm{~mW}$ and an integration time of $3 \mathrm{~s}$ for the Raman shifts of 300-1700 $\mathrm{cm}^{-1}$. Background-subtracted SERS spectra of the aromatic amino acids are shown in Figure 2 for the phenylalanine derivatives and in Figure 3 for the tyrosine derivatives.

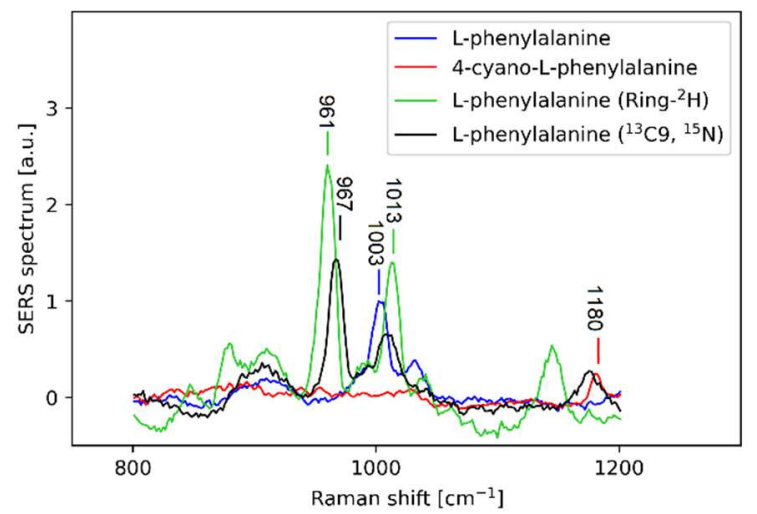

Fig. 2. SERS spectra of phenylalanine derivatives in the spectral region $800-1300 \mathrm{~cm}^{-1}$. The spectra are normalized on the 1003 $\mathrm{cm}^{-1}$ phenylalanine peak and most prominent peaks denoted.

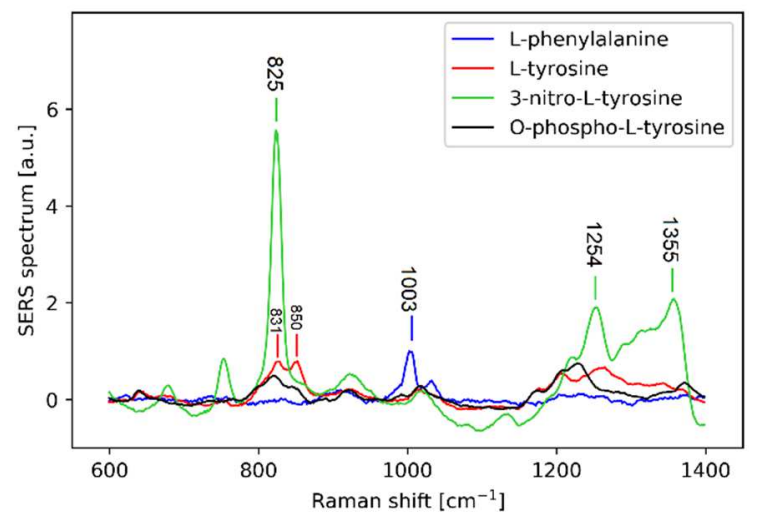

Fig. 3. SERS spectra of selected tyrosine derivatives in the spectral region $600-1400 \mathrm{~cm}^{-1}$. The SERS spectrum of phenylalanine is included for comparison with Figure 2, and the spectra are normalized on the $1003 \mathrm{~cm}^{-1}$ phenylalanine peak.

After subtracting the SERS background from the spectra, we identified SERS peaks pertaining to the investigated aromatics (Table 1). For our analysis we assumed that aromatic amino acid residues provide the dominant features in the SERS spectra of peptides [6].

Most of the examined aromatic amino acids provided characteristic SERS peaks in the examined spectral region, more specifically in the $600-1400 \mathrm{~cm}^{-1}$ range. We noticed that the SERS peaks of some aromatics overlap, so special care has to be taken to choose compatible aromatics as Raman reporters during multiplexing experiments. We additionally noticed that several non-natural aromatics provide strong SERS peaks compared to their corresponding natural aromatic amino acids, thus improving the signal-to-noise ratio. Of the examined non-natural aromatics, 3-nitro-tyrosine and the two phenylalanine molecules containing stable heavy isotopes seem particularly promising for multiplexing applications.

Table 1. Measured prominent SERS peak positions of different aromatic amino acids with relative SERS peak strengths. Abbreviations: vs: very strong, s: strong, m: medium, w: weak.

\begin{tabular}{|c|c|}
\hline Aromatic amino acid & $\begin{array}{c}\text { SERS peak position }\left[\mathrm{cm}^{-1}\right] \\
\text { with relative SERS peak } \\
\text { strength } \\
\end{array}$ \\
\hline L-phenylalanine & $1003(\mathrm{~s}), 1031(\mathrm{~m})$ \\
\hline L-tyrosine & $\begin{array}{l}831(\mathrm{~m}), 850(\mathrm{~m}), 1260 \\
(\mathrm{w})\end{array}$ \\
\hline L-tryptophan & $\begin{array}{l}754(\mathrm{~m}), 1118(\mathrm{w}), 1356 \\
(\mathrm{~m})\end{array}$ \\
\hline $\begin{array}{c}\text { 4-cyano- } \\
\text { L-phenylalanine }\end{array}$ & $1180(\mathrm{~m})$ \\
\hline $\begin{array}{l}\text { 4-benzoyl-L- } \\
\text { phenylalanine }\end{array}$ & $\begin{array}{l}1003(\mathrm{~m}), 1031(\mathrm{~m}), 1152 \\
(\mathrm{~m})\end{array}$ \\
\hline $\begin{array}{l}\text { 3,4-dichloro-L- } \\
\text { phenylalanine }\end{array}$ & - \\
\hline $\begin{array}{l}\text { L-phenylalanine } \\
\quad\left(\text { Ring- }^{2} \mathrm{H}\right)\end{array}$ & $\begin{array}{l}961(\mathrm{vs}), 1013(\mathrm{~s}), 1145 \\
(\mathrm{~s})\end{array}$ \\
\hline $\begin{array}{l}\text { L-phenylalanine } \\
\left({ }^{13} \mathrm{C} 9,{ }^{15} \mathrm{~N}\right)\end{array}$ & $\begin{array}{l}967(\mathrm{~s}), 1009(\mathrm{~m}), 1173 \\
(\mathrm{~m})\end{array}$ \\
\hline 3-nitro-L-tyrosine & $\begin{array}{l}752(\mathrm{~m}), 825(\mathrm{vs}), 1254 \\
(\mathrm{~s}), 1355(\mathrm{~s})\end{array}$ \\
\hline O-phospho-L-tyrosine & $\begin{array}{l}820(w), 843(w), 1229 \\
(w)\end{array}$ \\
\hline 3-amino-L-tyrosine & $\begin{array}{l}\text { Strong, but unstable SERS } \\
\text { signal }\end{array}$ \\
\hline
\end{tabular}

We have already successfully incorporated cyanophenylalanine into a peptide substrate and showed peptide cleavage by the trypsin protease [7]. In the future, we intend to incorporate the more promising non-natural aromatic amino acids into peptide substrates to demonstrate multiplexing capabilities of SERS detection of the protease activity.

Nina Turk acknowledges Research Foundation Flanders (FWO) for the predoctoral fellowship (1179319N).

\section{References}

1. P.C. Wuytens, H. Demol, N. Turk, K. Gevaert, A.G. Skirtach, M. Lamkanfi, R. Baets, Faraday Discuss, 205 (2017)

2. M. Drag, G.S. Salvesen, Nat Rev Drug Discov, 9 (2010)

3. J. Moger, P. Gribbon, A. Sewing, C.P. Winlove, Biochim Biophys Acta, 1770 (2007)

4. C.L. Weeks, A. Polishchuk, Z. Getahun, W.F. DeGrado, T.G. Spiro, J Raman Spectrosc, 39 (2008)

5. H.J. van Manen, A. Lenferink, C. Otto, Anal Chem, 80 (2008)

6. F. Wei, D. Zhang, N.J. Halas, J.D. Hartgerink, J Phys Chem B, 112 (2008)

7. N. Turk, A. Raza, P.C. Wuytens, H. Demol, A. Skirtach, K. Gevaert, R. Baets, 23rd Annual Symposium of the IEEE Photonics Benelux (2018) 